\title{
Derivation of capture and reaction cross sections from experi- mental quasi-elastic and elastic backscattering probabilities
}

\author{
V.V.Sargsyan ${ }^{1,2}$, G.G.Adamian ${ }^{1, a}$, N.V.Antonenko ${ }^{1}$, and P.R.S.Gomes ${ }^{3}$ \\ ${ }^{1}$ Joint Institute for Nuclear Research, 141980 Dubna, Russia \\ ${ }^{2}$ International Center for Advanced Studies, Yerevan State University, 0025 Yerevan, Armenia \\ ${ }^{3}$ Instituto de Fisica, Universidade Federal Fluminense, Av. Litorânea, s/n, Niterói, R.J. 24210-340, Brazil
}

\begin{abstract}
We suggest simple and useful methods to extract reaction and capture (fusion) cross sections from the experimental elastic and quasi-elastic backscattering data.
\end{abstract}

\section{Introduction}

The direct measurement of the reaction or capture (fusion) cross section is a difficult task since it would require the measurement of individual cross sections of many reaction channels, and most of them could be reached only by specific experiments. This would require different experimental setups not always available at the same laboratory and, consequently, such direct measurements would demand a large amount of beam time and would take probably some years to be reached. Because of that, the measurements of elastic scattering angular distributions that cover full angular ranges and optical model analysis have been used for the determination of reaction cross sections. This traditional method consists in deriving the parameters of the complex optical potentials which fit the experimental elastic scattering angular distributions and then of deriving the reaction cross sections predicted by these potentials. Even so, both the experimental part and the analysis of this latter method are not so simple. In the present work we present a much simpler method to determine reaction and capture (fusion) cross sections. It consists of measuring only elastic or quasi-elastic scattering at one backward angle, and from that, the extraction of the reaction or capture cross sections can easily be performed.

\section{Relationship between capture and quasi-elastic backscattering, and relationship between reaction and elastic backscattering}

From the conservation of the total reaction flux one can write [1-4] the expressions

$$
P_{e l}\left(E_{\text {c.m. }}, J\right)+P_{R}\left(E_{\text {c.m. }}, J\right)=1
$$

and

$$
P_{q e}\left(E_{\mathrm{c} . \mathrm{m} .}, J\right)+P_{c a p}\left(E_{\mathrm{c} . \mathrm{m} .}, J\right)+P_{B U}\left(E_{\mathrm{c} . \mathrm{m} .}, J\right)+P_{D I C}\left(E_{\mathrm{c} . \mathrm{m} .}, J\right)=1 .
$$

\footnotetext{
a e-mail: adamian@ theor.jinr.ru
} 
The quasi-elastic scattering probability

$$
P_{q e}\left(E_{\mathrm{c} . \mathrm{m} .}, J\right)=P_{e l}\left(E_{\mathrm{c} . \mathrm{m} .}, J\right)+P_{i n}\left(E_{\mathrm{c} . \mathrm{m} .}, J\right)+P_{t r}\left(E_{\mathrm{c} . \mathrm{m} .}, J\right)
$$

is defined as the sum of elastic scattering $P_{e l}$, inelastic excitations $P_{i n}$ and few-nucleon transfer $P_{t r}$ probabilities. The reaction probability may be written as

$$
P_{R}\left(E_{\text {c.m. }}, J\right)=P_{i n}\left(E_{\text {c.m. }}, J\right)+P_{t r}\left(E_{\text {c.m. }}, J\right)+P_{c a p}\left(E_{\text {c.m. }}, J\right)+P_{B U}\left(E_{\text {c.m. }}, J\right)+P_{D I C}\left(E_{\text {c.m. }}, J\right),
$$

where $P_{c a p}$ is the capture probability (sum of evaporation-residue formation, fusion-fission, and quasifission probabilities or sum of fusion and quasi-fission probabilities), $P_{D I C}$ is the deep inelastic collision probability, and $P_{B U}$ is the breakup probability, important particularly when weakly bound nuclei are involved in the reaction [4]. Note that the deep inelastic collision process is only important at large energies above the Coulomb barrier. Here we neglect the deep inelastic collision process, since we are concerned with low energies. Thus, one can extract the reaction

$$
P_{R}^{e x}\left(E_{\mathrm{c} . \mathrm{m} .}, J=0\right)=1-P_{e l}\left(E_{\mathrm{c} . \mathrm{m} .}, J=0\right)
$$

and capture

$$
P_{c a p}^{e x}\left(E_{\mathrm{c} . \mathrm{m} .}, J=0\right)=1-\left[P_{q e}\left(E_{\mathrm{c} . \mathrm{m} .}, J=0\right)+P_{B U}\left(E_{\mathrm{c} . \mathrm{m} .}, J=0\right)\right]
$$

probabilities at $J=0$ from the experimental elastic backscattering probability $P_{e l}\left(E_{\text {c.m. }}, J=0\right)$ and quasi-elastic backscattering probability $P_{q e}\left(E_{\mathrm{c} . \mathrm{m}}, J=0\right)$ plus breakup probability $P_{B U}\left(E_{\mathrm{c} . \mathrm{m} .}, J=0\right)$ at backward angle, respectively. Here, the elastic or quasi-elastic scattering or breakup probability [4-8]

$$
P_{e l, q e, B U}\left(E_{\mathrm{c} . \mathrm{m} .}, J=0\right)=d \sigma_{e l, q e, B U} / d \sigma_{R u}
$$

for angular momentum $J=0$ is given by the ratio of the elastic or quasi-elastic scattering or breakup differential cross section and Rutherford differential cross section at 180 degrees. Furthermore, one can approximate the $J$ dependence of the reaction $P_{R}\left(E_{\text {c.m. }}, J\right)$ and capture $P_{c a p}\left(E_{\text {c.m. }}, J\right)$ probabilities at a given bombarding energy $E_{\mathrm{c} . \mathrm{m}}$. by shifting the energy [1,2]:

$$
P_{R}\left(E_{\mathrm{c} . \mathrm{m} .}, J\right) \approx P_{R}^{e x}\left(E_{\mathrm{c} . \mathrm{m} .}-\frac{\hbar^{2} \Lambda}{2 \mu R_{b}^{2}}-\frac{\hbar^{4} \Lambda^{2}}{2 \mu^{3} \omega_{b}^{2} R_{b}^{6}}, J=0\right)
$$

and

$$
P_{c a p}\left(E_{\text {c.m. }}, J\right) \approx P_{c a p}^{e x}\left(E_{\text {c.m. }}-\frac{\hbar^{2} \Lambda}{2 \mu R_{b}^{2}}-\frac{\hbar^{4} \Lambda^{2}}{2 \mu^{3} \omega_{b}^{2} R_{b}^{6}}, J=0\right),
$$

where $\Lambda=J(J+1), R_{b}=R_{b}(J=0)$ is the position of the Coulomb barrier at $J=0, \mu=m_{0} A_{1} A_{2} /\left(A_{1}+\right.$ $A_{2}$ ) is the reduced mass ( $m_{0}$ is the nucleon mass), and $\omega_{b}$ is the curvature of the s-wave potential barrier. Here we used the expansion of the height $V_{b}(J)$ of the Coulomb barrier up to second order in $\Lambda[1,2]$ :

$$
V_{b}(J)=V_{b}(J=0)+\frac{\hbar^{2} \Lambda}{2 \mu R_{b}^{2}}+\frac{\hbar^{4} \Lambda^{2}}{2 \mu^{3} \omega_{b}^{2} R_{b}^{6}} .
$$

Employing formulas for the reaction

$$
\sigma_{R}\left(E_{\text {c.m. }}\right)=\pi \hbar^{2} \sum_{J=0}^{\infty}(2 J+1) P_{R}^{e x}\left(E_{\text {c.m. }}-\frac{\hbar^{2} \Lambda}{2 \mu R_{b}^{2}}-\frac{\hbar^{4} \Lambda^{2}}{2 \mu^{3} \omega_{b}^{2} R_{b}^{6}}, J=0\right)
$$


and capture

$$
\sigma_{c a p}\left(E_{\text {c.m. }}\right)=\pi \lambda^{2} \sum_{J=0}^{J_{c r}}(2 J+1) P_{c a p}^{e x}\left(E_{\text {c.m. }}-\frac{\hbar^{2} \Lambda}{2 \mu R_{b}^{2}}-\frac{\hbar^{4} \Lambda^{2}}{2 \mu^{3} \omega_{b}^{2} R_{b}^{6}}, J=0\right)
$$

cross sections, converting the sum over the partial waves $J$ into an integral, and expressing $J$ by the variable $E=E_{\text {c.m. }}-\frac{\hbar^{2} \Lambda}{2 \mu R_{b}^{2}}$, we obtain the following simple expressions $[1,2]$ :

$$
\sigma_{R}\left(E_{\mathrm{c} . \mathrm{m} .}\right)=\frac{\pi R_{b}^{2}}{E_{\mathrm{c} . \mathrm{m} .}} \int_{0}^{E_{\mathrm{c} . \mathrm{m} .}} d E P_{R}^{e x}(E, J=0)\left[1-\frac{4\left(E_{\mathrm{c} . \mathrm{m} .}-E\right)}{\mu \omega_{b}^{2} R_{b}^{2}}\right]
$$

and

$$
\sigma_{c a p}\left(E_{\text {c.m. }}\right)=\frac{\pi R_{b}^{2}}{E_{\text {c.m. }}} \int_{E_{\text {c.m. }-}-\frac{\hbar^{2} \Lambda c r}{2 \mu R_{b}^{2}}}^{E_{\text {c.m. }}} d E P_{c a p}^{e x}(E, J=0)\left[1-\frac{4\left(E_{\text {c.m. }}-E\right)}{\mu \omega_{b}^{2} R_{b}^{2}}\right],
$$

where $\lambda^{2}=\hbar^{2} /\left(2 \mu E_{\text {c.m. }}\right)$ is the reduced de Broglie wavelength, $\Lambda_{c r}=J_{c r}\left(J_{c r}+1\right)$, and $J=J_{c r}$ is the critical angular momentum. For values $J$ greater than $J_{c r}$, the potential pocket in the nucleus-nucleus interaction potential vanishes and capture does not occur. To calculate the critical angular momentum $J_{c r}$ and the position $R_{b}$ of the Coulomb barrier, we use the nucleus-nucleus interaction potential $V(R, J)$ of Ref. [9]. For the nuclear part of the nucleus-nucleus potential, the double-folding formalism with the Skyrme-type density-dependent effective nucleon-nucleon interaction is employed [9].

For systems with $Z_{1} \times Z_{2}<2000\left(Z_{1,2}\right.$ are the atomic numbers of interacting nuclei), the critical angular momentum $J_{c r}$ is large enough and Eq. (13) can be approximated with good accuracy as:

$$
\sigma_{\text {cap }}\left(E_{\text {c.m. }}\right) \approx \frac{\pi R_{b}^{2}}{E_{\text {c.m. }}} \int_{0}^{E_{\text {c.m. }}} d E P_{c a p}^{e x}(E, J=0)\left[1-\frac{4\left(E_{\mathrm{c} . \mathrm{m} .}-E\right)}{\mu \omega_{b}^{2} R_{b}^{2}}\right] .
$$

The formula (12) [(13)] relates the reaction [capture] cross section with the elastic [quasi-elastic] scattering excitation function at a backward angle. By using the experimental $P_{e l}\left(E_{\text {c.m. }}, J=0\right)$ $\left[P_{q e}\left(E_{\text {c.m. }}, J=0\right)+P_{B U}\left(E_{\text {c.m. }}, J=0\right)\right]$ and Eq. (12) [(13)], one can obtain the reaction [capture] cross section.

It is important to mention that since the generalized form of the optical theorem connects the reaction cross section and forward elastic scattering amplitude[4], we show that the forward and backward elastic scattering amplitudes are related to each other.

Using the extracted $\sigma_{c a p}$ and the experimental $P_{q e}$, one can find the average angular momentum

$$
\begin{aligned}
<J>=\frac{\pi R_{b}^{2}}{E_{\text {c.m. } .} \sigma_{c a p}\left(E_{\text {c.m. }}\right)} \int_{E_{\text {c.m. } .}-\frac{\hbar^{2} \Lambda_{c r}}{2 \mu R_{b}^{2}}}^{E_{\text {c.m. }}} & d E P_{\text {cap }}^{e x}(E, J=0)\left[1-\frac{5\left(E_{\mathrm{c} . \mathrm{m} .}-E\right)}{\mu \omega_{b}^{2} R_{b}^{2}}\right] \\
& \times\left[\left(\frac{2 \mu R_{b}^{2}}{\hbar^{2}}\left(E_{\text {c.m. }}-E\right)+\frac{1}{4}\right)^{1 / 2}-\frac{1}{2}\right]
\end{aligned}
$$

and the second moment of the angular momentum

$$
\begin{aligned}
& <J(J+1)>=\frac{2 \pi \mu R_{b}^{4}}{\hbar^{2} E_{\text {c.m. } . ~} \sigma_{c a p}\left(E_{\text {c.m. }}\right)} \int_{E_{\text {c.m. }}-\frac{\hbar^{2} \Lambda c r}{2 \mu R_{b}^{2}}}^{E_{\text {c.m. }}} \quad d E P_{c a p}^{e x}(E, J=0)\left[1-\frac{6\left(E_{\text {c.m. }}-E\right)}{\mu \omega_{b}^{2} R_{b}^{2}}\right] \\
& \times\left[E_{\mathrm{c} . \mathrm{m} .}-E\right]
\end{aligned}
$$

of the captured system [1]. 


\section{Results of calculations}

As the elastic, quasi-elastic, and breakup data were not taken at 180 degrees, but rather at backward angles in the range from 150 to 170 degrees, the corresponding center of mass energies were corrected by the centrifugal potential at the experimental angle [5].
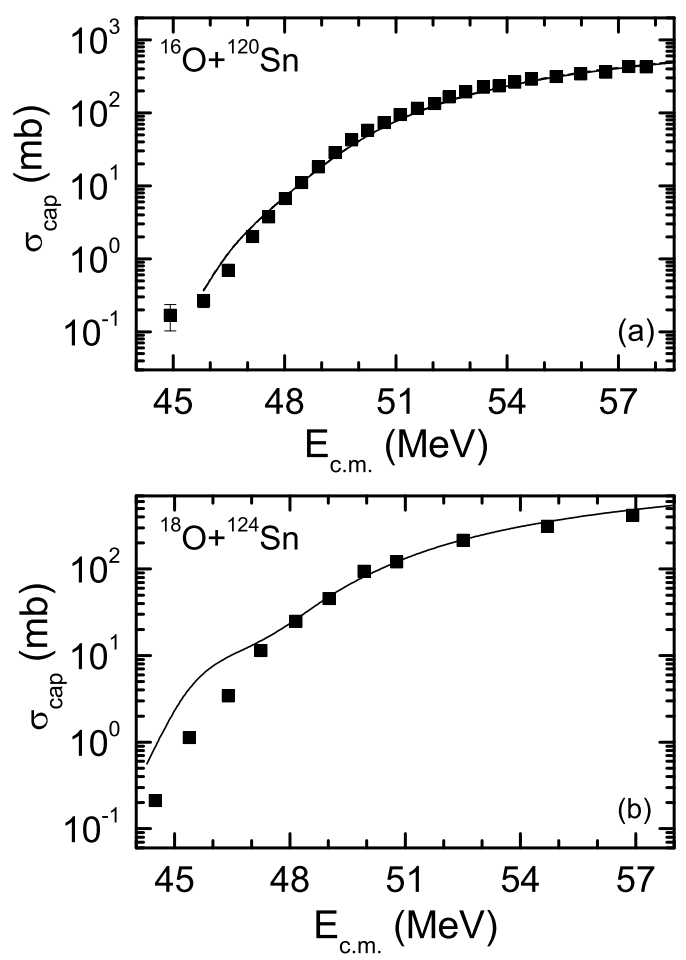

Figure 1. The extracted capture cross sections for the reactions ${ }^{16} \mathrm{O}+{ }^{120} \mathrm{Sn}(\mathrm{a})$ and ${ }^{18} \mathrm{O}+{ }^{124} \mathrm{Sn}(\mathrm{b})$ by employing Eq. (13) (solid line) and Eq. (14) (dotted line). These lines almost coincide. The experimental quasi-elastic backscattering data are from Ref. [10]. The experimental capture (fusion) data (symbols) are from Refs. [10, 11].

\subsection{Capture cross sections}

For the verification of our method of the extraction of $\sigma_{c a p}$, first we compare the extracted capture cross sections with experimental ones for the reactions with tightly bound nuclei $\left[P_{B U}\left(E_{\text {c.m. }}, J=0\right)=\right.$ 0]. In Figs. 1 and 2 one can see good agreement between the extracted and directly measured capture cross sections for the reactions ${ }^{16} \mathrm{O}+{ }^{120} \mathrm{Sn},{ }^{18} \mathrm{O}+{ }^{124} \mathrm{Sn},{ }^{16} \mathrm{O}+{ }^{208} \mathrm{~Pb}$, and ${ }^{16} \mathrm{O}+{ }^{144} \mathrm{Sm}$ at energies above the Coulomb barrier. The results in the sub-barrier energy region are discussed later. To extract the capture cross section, we use both Eq. (13) (solid lines) and Eq. (14) (dotted lines). The values of critical angular momentum used are $J_{c r}=54,56,57$, and 62 for the reactions ${ }^{16} \mathrm{O}+{ }^{120} \mathrm{Sn},{ }^{18} \mathrm{O}+$ ${ }^{124} \mathrm{Sn},{ }^{16} \mathrm{O}+{ }^{144} \mathrm{Sm}$, and ${ }^{16} \mathrm{O}+{ }^{208} \mathrm{~Pb}$, respectively. The difference between the results of Eqs. (13) and (14) is less than 5\% at the highest energies. At low energies, Eqs. (13) and (14) lead to the same values of $\sigma_{c a p}$. The factor $1-\frac{4\left(E_{\text {c.m. }}-E\right)}{\mu \omega_{b}^{2} R_{b}^{2}}$ in Eqs. (13) and (14) very weakly influences the results of the calculations for the systems and energies considered. Hence, one can say that for the relatively light systems the proposed method of extracting the capture cross section is model independent (in particular, independent of the potential used):

$$
\sigma_{c a p}\left(E_{\mathrm{c} . \mathrm{m} .}\right) \approx \frac{\pi R_{b}^{2}}{E_{\mathrm{c} . \mathrm{m} .}} \int_{0}^{E_{\mathrm{c} . \mathrm{m} .}} d E P_{\text {cap }}^{e x}(E, J=0) .
$$



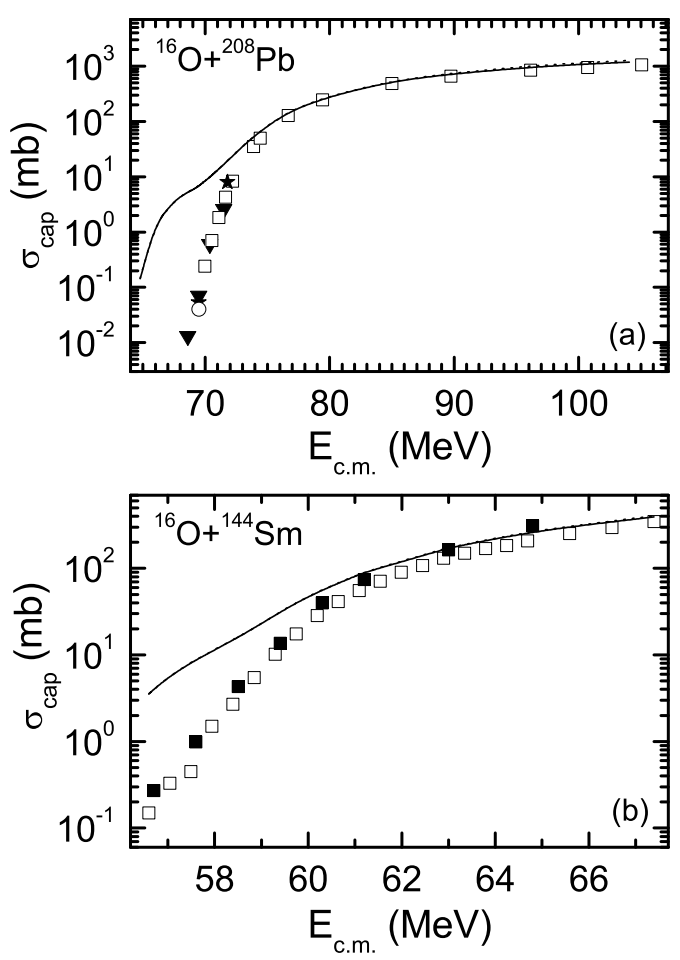

Figure 2. The same as in Fig. 1, but for the reactions ${ }^{16} \mathrm{O}+{ }^{208} \mathrm{~Pb}(\mathrm{a}),{ }^{144} \mathrm{Sm}(\mathrm{b})$. The experimental quasi-elastic backscattering data are from Refs. [5, 7]. For the ${ }^{16} \mathrm{O}+{ }^{208} \mathrm{~Pb}$ reaction, the experimental capture (fusion) data are from Refs. [12] (open squares), [13] (open circles), [14] (closed stars), and [15] (closed triangles). For the ${ }^{16} \mathrm{O}+{ }^{144} \mathrm{Sm}$ reaction, the experimental capture (fusion) data are from Refs. [16] (closed squares) and [17] (open squares).

One can see that the formulas used are suitable not only for almost spherical nuclei (Figs. 1 and 2) but also for reactions with strongly deformed target- or projectile-nuclei (Figs. 3 and 4). So, the deformation effect is effectively contained in the experimental $P_{q e}$. One finds $J_{c r}=58,68,74$, and 76 for the reactions ${ }^{16} \mathrm{O}+{ }^{154} \mathrm{Sm},{ }^{32} \mathrm{~S}+{ }^{90} \mathrm{Zr},{ }^{32} \mathrm{~S}+{ }^{96} \mathrm{Zr}$, and ${ }^{20} \mathrm{Ne}+{ }^{208} \mathrm{~Pb}$, respectively. The results obtained by employing Eq. (14) are almost the same and not presented in Figs. 3 and 4.

For the reactions ${ }^{16} \mathrm{O}+{ }^{154} \mathrm{Sm}$ and ${ }^{32} \mathrm{~S}+{ }^{96} \mathrm{Zr}$, the extracted capture cross sections are shifted in energy by 1.7 and $1.9 \mathrm{MeV}$ with respect to the measured capture data, respectively. This could be the result of different energy calibrations in the experiments on the capture measurement and on the quasi-elastic scattering. Because of the lack of systematics in these energy shifts, their origin remains unclear and we adjust the Coulomb barriers in the extracted capture cross sections to the values following the experiments.

Note that the extracted and experimental capture cross sections deviate from each other in the reactions ${ }^{16} \mathrm{O}+{ }^{208} \mathrm{~Pb},{ }^{16} \mathrm{O}+{ }^{144} \mathrm{Sm}$, and ${ }^{32} \mathrm{~S}+{ }^{90} \mathrm{Zr}$ at energies below the Coulomb barrier. Probably this deviation (the mismatch between quasi-elastic backscattering and fusion (capture) experimental data) is a reason for the large discrepancies in the diffuseness parameter extracted from the analyses of the quasi-elastic scattering and fusion (capture) at deep sub-barrier energies. One of the possible reasons for the overestimation of the capture cross section from the quasi-elastic data at sub-barrier energies is the underestimation of the total reaction differential cross section taken as the Rutherford differential cross section. Indeed, for the ${ }^{32} \mathrm{~S}+{ }^{90} \mathrm{Zr}$ reaction, the increase of $P_{q e}$ within $2-3 \%$ is needed in order to obtain the agreement between the extracted and measured capture cross sections at the sub-barrier energies [Fig. 4(a)].

As seen in Fig. 5, the extracted capture cross sections $\sigma_{c a p}\left(E_{\text {c.m. }}\right)$ (solid line) for the ${ }^{6} \mathrm{Li}+{ }^{208} \mathrm{~Pb}$ reaction with weakly bound nucleus $\left[P_{B U}\left(E_{\mathrm{c} . \mathrm{m} .}, J=0\right) \neq 0\right]$ are rather close to those found in the 

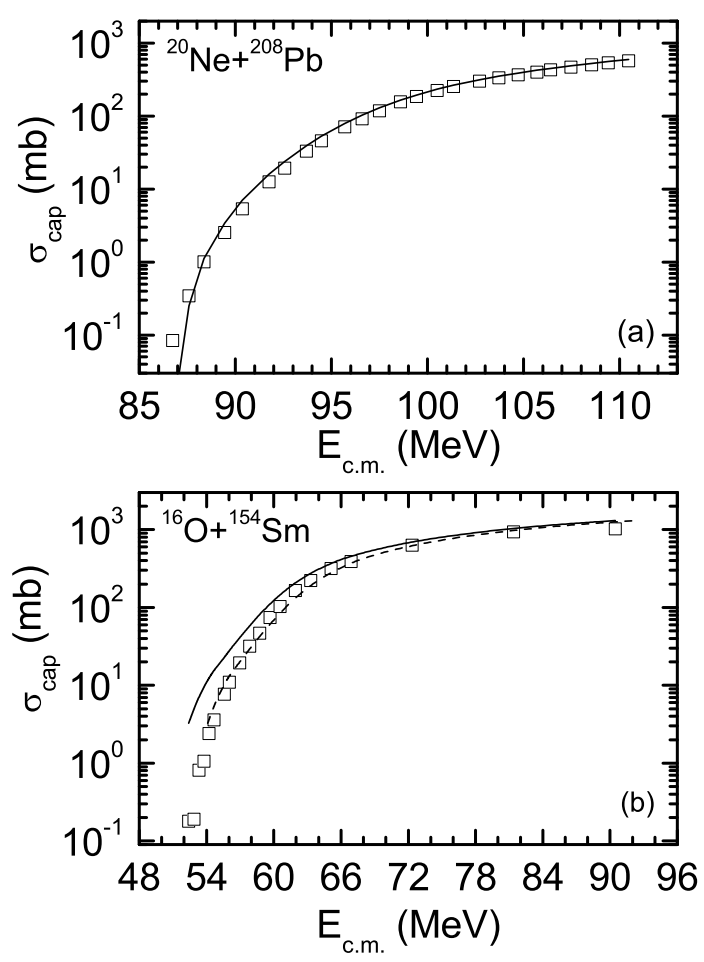

Figure 3. The same as in Fig. 1, but for the reactions ${ }^{20} \mathrm{Ne}+{ }^{208} \mathrm{~Pb}$ and ${ }^{16} \mathrm{O}+{ }^{154} \mathrm{Sm}$. The experimental quasi-elastic backscattering data are from Refs. [5, 18]. The experimental capture (fusion) data (symbols) are from Refs. [17, 18]. For the ${ }^{16} \mathrm{O}+$ ${ }^{154} \mathrm{Sm}$ reaction, the dashed line is obtained from the shift of the solid line by $1.7 \mathrm{MeV}$ to higher energies.

direct measurements [24] at energies above the Coulomb barrier. It appears that at energies near and below the Coulomb barrier the extracted $\sigma_{c a p}\left(E_{\text {c.m. }}\right)$ deviates from the direct measurements. It is similarly possible to calculate the capture excitation function

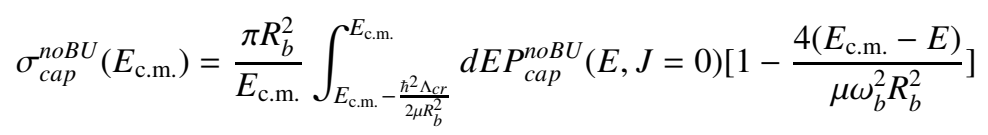

in the absence of the breakup process (Fig. 5, dotted line) by using the following formula for the capture probability in this case [23]:

$$
P_{c a p}^{n o B U}\left(E_{\mathrm{c} . \mathrm{m} .}, J=0\right)=1-\frac{P_{q e}\left(E_{\mathrm{c} . \mathrm{m} .}, J=0\right)}{1-P_{B U}\left(E_{\mathrm{c} . \mathrm{m} .}, J=0\right)} .
$$

By employing the measured excitation functions $P_{q e}$ and $P_{B U}$ at the backward angle [22], Eqs. (13), (17), and the formula

$$
<P_{B U}>\left(E_{\mathrm{c} . \mathrm{m} .}\right)=1-\frac{\sigma_{c a p}\left(E_{\mathrm{c} . \mathrm{m} .}\right)}{\sigma_{c a p}^{\text {noBU }}\left(E_{\mathrm{c} . \mathrm{m} .}\right)},
$$

we extract the mean breakup probability $<P_{B U}>\left(E_{\text {c.m. }}\right.$ ) averaged over all partial waves $J$ (Fig. 6). The value of $\left\langle P_{B U}>\right.$ has a maximum at $E_{\text {c.m. }}-V_{b} \approx 4 \mathrm{MeV}\left(<P_{B U}>=0.26\right)$ and slightly (sharply) decreases with increasing (decreasing) $E_{\text {c.m. }}$. The experimental breakup excitation function at backward angle has similar energy behavior [22]. By comparing the calculated capture cross sections in the absence of breakup and experimental capture (complete fusion) data, the opposite energy trend is found in Ref. [23], where $<P_{B U}>$ has a minimum at $E_{\text {c.m. }}-V_{b} \approx 2 \mathrm{MeV}\left(<P_{B U}>=0.34\right)$ and 

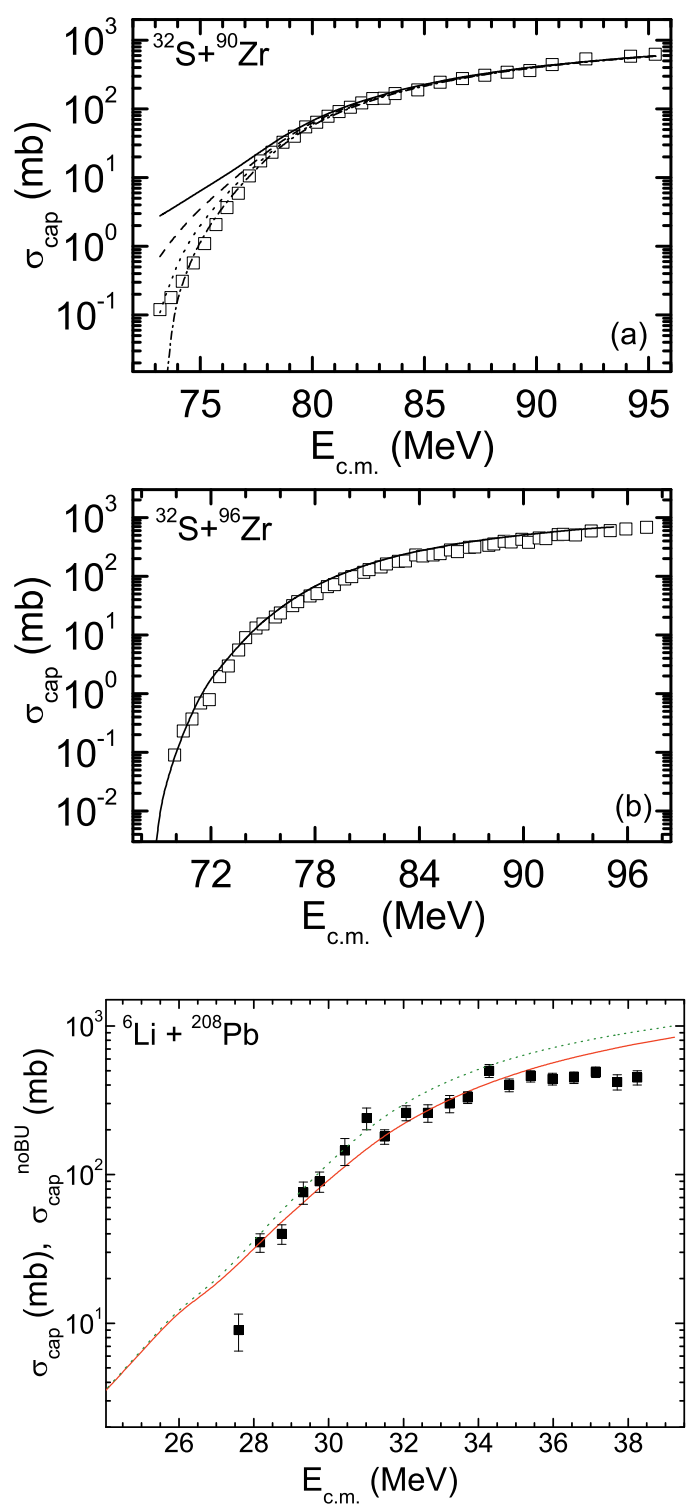

Figure 4. The same as in Fig. 1, but for the reactions ${ }^{32} \mathrm{~S}$ $+{ }^{90} \mathrm{Zr}$ (a) and ${ }^{32} \mathrm{~S}+{ }^{96} \mathrm{Zr}(\mathrm{b})$. For the ${ }^{32} \mathrm{~S}+{ }^{90} \mathrm{Zr}$ reaction, we show the extracted capture cross sections, increasing the experimental $P_{q e}$ by $1 \%$ (dashed line), $2 \%$ (dotted line), and 3\% (dash-dotted line). The experimental quasi-elastic backscattering data are from Ref. [19]. The experimental capture (fusion) data (symbols) are from Ref. [20]. For the ${ }^{32} \mathrm{~S}+{ }^{96} \mathrm{Zr}$ reaction. The energy scale for the extracted capture cross sections is adjusted to that of the direct measurements.
Figure 5. (Color online) The extracted capture cross sections $\sigma_{\text {cap }}\left(E_{\mathrm{c} . \mathrm{m} .}\right)$ (solid line) and $\sigma_{\text {cap }}^{\text {noBU }}\left(E_{\mathrm{c} . \mathrm{m} .}\right.$ ) (dotted line) for the ${ }^{6} \mathrm{Li}+{ }^{208} \mathrm{~Pb}$ reaction. The used experimental quasi-elastic backscattering and quasi-elastic backscattering plus breakup data at the backward angle are from Ref. [22]. The experimental capture cross sections (solid squares) are from Ref. [24]. The energy scale for the extracted capture cross sections is adjusted to that of the direct measurements.

globally increases in both sides of this minimum. It is also shown in Refs. [23, 25] that there are no systematic trends of breakup in the complete fusion reactions with the light projectiles ${ }^{9} \mathrm{Be},{ }^{6,7,9} \mathrm{Li}$, and ${ }^{6,8} \mathrm{He}$ at near-barrier energies. Thus, by employing the experimental quasi-elastic backscattering, one can obtain additional information about the breakup process. By using Eqs. (15) and (16) and the experimental $P_{q e}$, we extract $\langle J\rangle$ and $\left\langle J^{2}\right\rangle$ of the captured system for the reactions ${ }^{16} \mathrm{O}+{ }^{154} \mathrm{Sm}$ and ${ }^{16} \mathrm{O}+{ }^{208} \mathrm{~Pb}$, respectively (Fig. 7). The agreement with the results of direct measurements of the $\gamma$-multiplicities in the corresponding complete fusion reactions is quite good. For the ${ }^{16} \mathrm{O}+{ }^{208} \mathrm{~Pb}$ reaction at sub-barrier energies, the difference between the extracted and experimental angular momenta is related with the deviation of the extracted capture excitation function from the experimental one (see Fig. 2). 

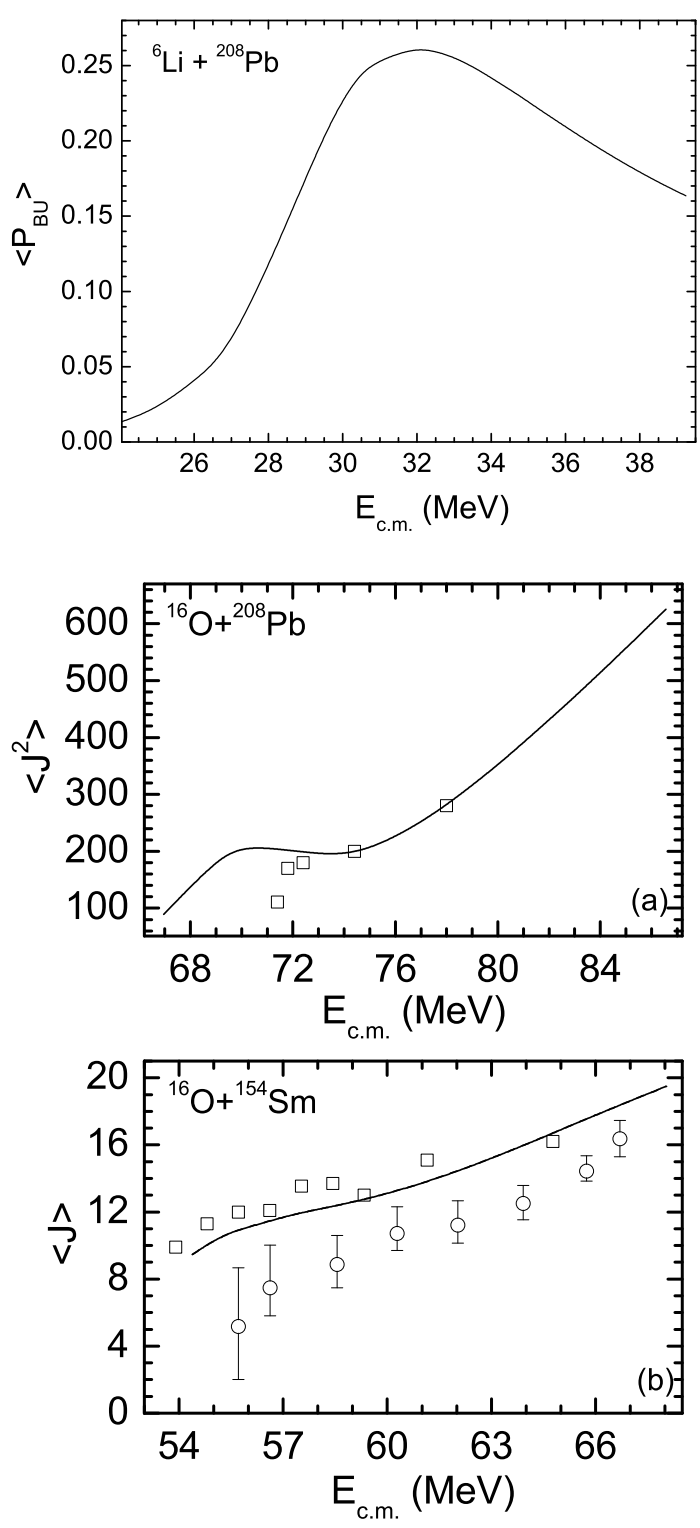

Figure 6. The extracted mean breakup probability $\left\langle P_{B U}>\left(E_{\text {c.m. }}\right)\right.$ [Eq. (19)] as a function of bombarding energy $E_{\text {c.m. }}$ for the ${ }^{6} \mathrm{Li}+{ }^{208} \mathrm{~Pb}$ reaction. The experimental quasi-elastic backscattering and quasi-elastic backscattering plus breakup data at the backward angle are from Ref. [22].

Figure 7. The extracted $\langle J\rangle$ and $\left\langle J^{2}\right\rangle$ for the reactions ${ }^{16} \mathrm{O}+{ }^{208} \mathrm{~Pb}$ (a) and ${ }^{16} \mathrm{O}+{ }^{154} \mathrm{Sm}$ (b) by employing Eqs. (15) and (16). The experimental quasi-elastic backscattering data are from Ref. [7]. The experimental data of $\left\langle J^{2}\right\rangle$ and $\langle J\rangle$ are from Refs. [26] (open squares) and [27, 28] (open squares and circles), respectively.

\subsection{Reaction cross sections}

As can be observed in Figs. 8-15, there is a good agreement between the reaction cross sections extracted from the experimental elastic scattering at backward angle and from the experimental elastic scattering angular distributions with optical potential for the reactions ${ }^{4} \mathrm{He}+{ }^{92} \mathrm{Mo},{ }^{4} \mathrm{He}+{ }^{110,116} \mathrm{Cd}$, ${ }^{4} \mathrm{He}+{ }^{112,120} \mathrm{Sn},{ }^{16} \mathrm{O}+{ }^{208} \mathrm{~Pb}$, and ${ }^{6,7} \mathrm{Li}+{ }^{64} \mathrm{Zn}$ at energies near and above the Coulomb barrier. One can see that the equation used, Eq. (12), is suitable not only for almost spherical nuclei, but also for the reactions with slightly deformed target-nuclei. The deformation effect is effectively contained in the experimental $P_{e l}$. For very deformed nuclei, it is not possible experimentally to separate elastic events from the low-lying inelastic excitations. In our calculations, to obtain better agreement for the 

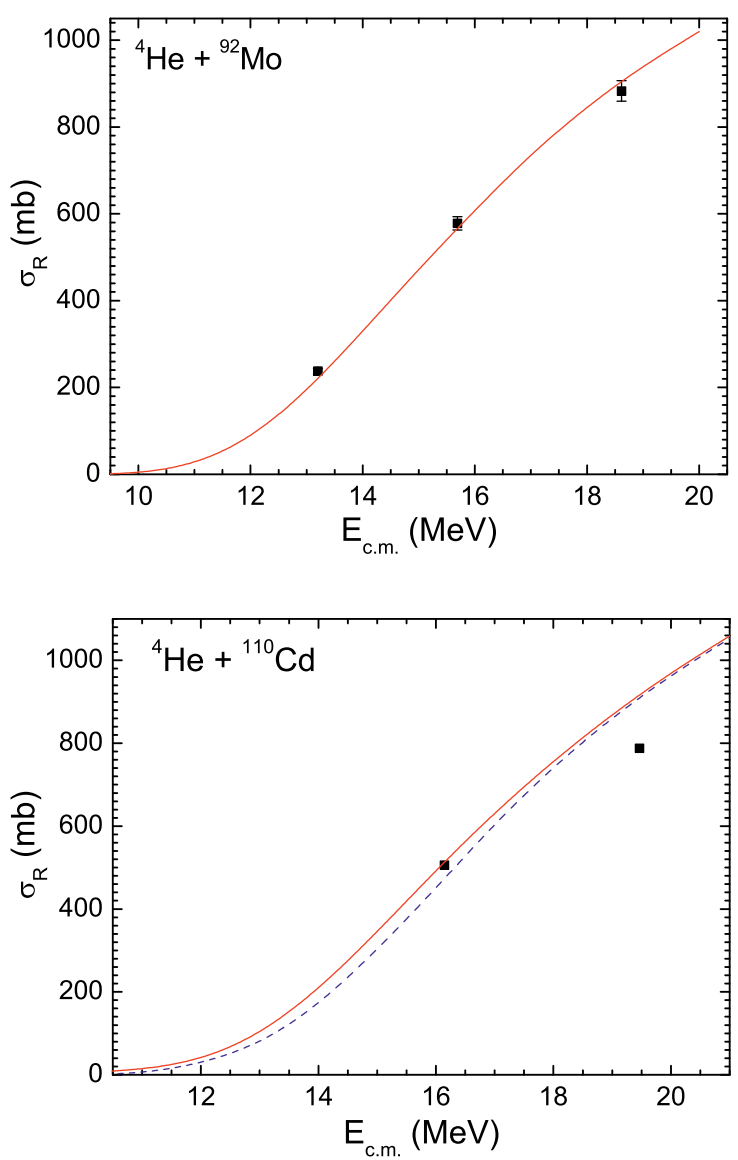

Figure 8. (Color online) The extracted reaction cross sections (solid line) for the ${ }^{4} \mathrm{He}+{ }^{92} \mathrm{Mo}$ reaction by employing Eq. (12). The experimental elastic scattering probabilities at the backward angle are from Ref. [29]. The reaction cross sections extracted from the experimental elastic scattering angular distribution with optical potential are presented by squares [29].

Figure 9. (Color online) The extracted reaction cross sections (lines) for the ${ }^{4} \mathrm{He}+{ }^{110} \mathrm{Cd}$ reaction by employing Eq. (12). The experimental elastic scattering probabilities at the backward angle are from Refs. [31, 32] (solid line) and Ref. [33] (dashed line). The reaction cross sections extracted from the experimental elastic scattering angular distribution with optical potential are presented by squares [29].

reactions ${ }^{16} \mathrm{O}+{ }^{208} \mathrm{~Pb}$ and ${ }^{6} \mathrm{Li}+{ }^{64} \mathrm{Zn}$, the extracted reaction cross sections were shifted in energy by 0.3 $\mathrm{MeV}$ to higher energies and $0.4 \mathrm{MeV}$ to lower energies with respect to the measured experimental data, respectively. There is no clear physical justification for the energy shift. The most probable reason might be related with the uncertainty associated with the elastic scattering data. By using Eq. (13), the capture cross sections of the reactions ${ }^{6,7} \mathrm{Li}+{ }^{64} \mathrm{Zn}$ can be extracted, if one assumes that $P_{B U}=0$, since it is much smaller than $P_{q e}$. In Figs. 14 and 15 we also show the results of our calculations for capture cross sections of the ${ }^{6,7} \mathrm{Li}+{ }^{64} \mathrm{Zn}$ systems, for which the fusion process can be considered to exhaust the capture cross section. Figure 14 shows that the extracted and experimental capture cross sections are in good agreement for the ${ }^{6} \mathrm{Li}+{ }^{64} \mathrm{Zn}$ reaction at energies near and above the Coulomb barrier for the data taken in Refs. [34, 35]. Note that the extracted capture excitation function is shifted in energy by $0.7 \mathrm{MeV}$ to higher energies with respect to the experimental data. This could be the result of different energy calibrations in the experiments on the capture measurement and quasi-elastic scattering.

For the ${ }^{7} \mathrm{Li}+{ }^{64} \mathrm{Zn}$ reaction, the $Q$-value of the one neutron stripping transfer is positive and this process should have a reasonable high probability to occur, whereas for the ${ }^{6} \mathrm{Li}+{ }^{64} \mathrm{Zn}$ reaction, $Q$ values of neutron transfers are negative. Therefore, one might expect that transfer cross sections for ${ }^{7} \mathrm{Li}+{ }^{64} \mathrm{Zn}$ are larger than for ${ }^{6} \mathrm{Li}+{ }^{64} \mathrm{Zn}$. With concern for breakup, since ${ }^{6} \mathrm{Li}$ has a smaller threshold energy for breakup than ${ }^{7} \mathrm{Li}$, one might expect that breakup cross sections for ${ }^{6} \mathrm{Li}+{ }^{64} \mathrm{Zn}$ are larger than 

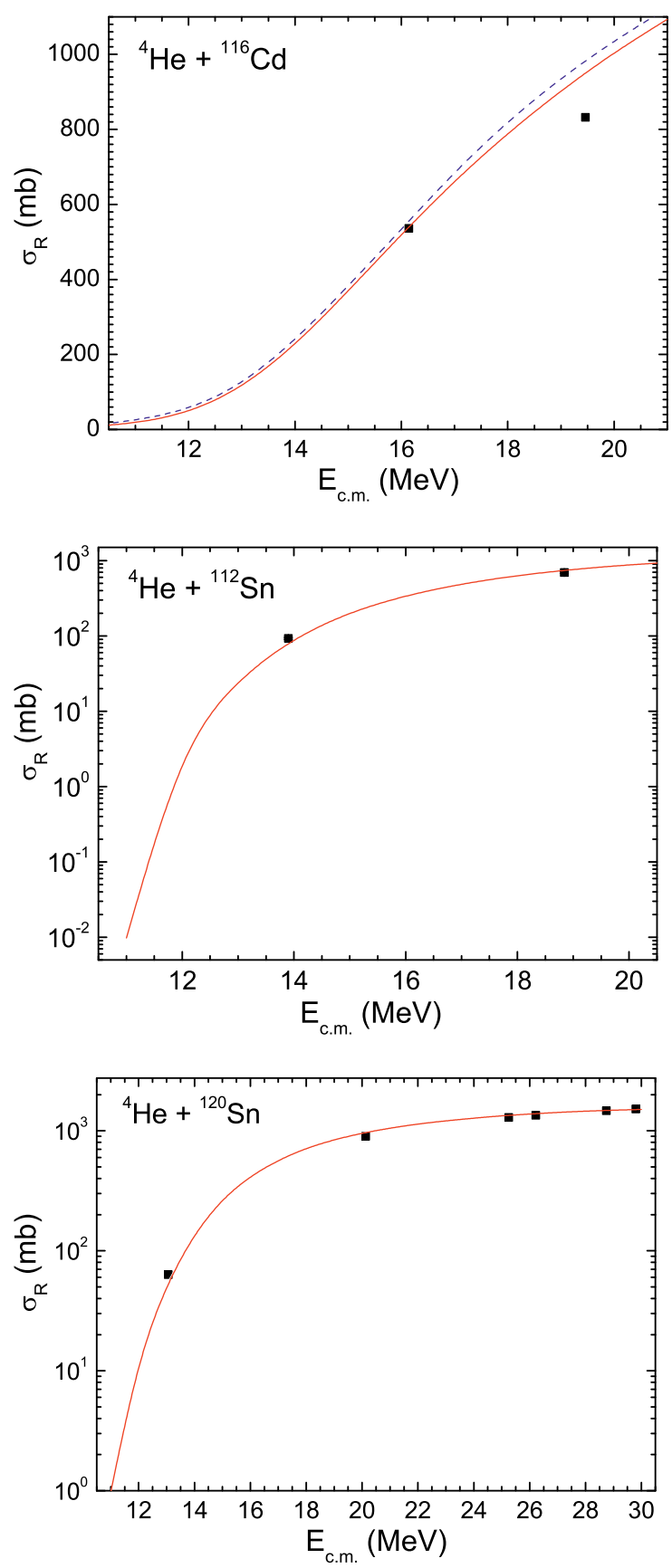

Figure 10. (Color online) The same as in Fig. 9, but for the ${ }^{4} \mathrm{He}+{ }^{116} \mathrm{Cd}$ reaction.

Figure 11. (Color online) The extracted reaction cross sections (solid line) for the ${ }^{4} \mathrm{He}+{ }^{112} \mathrm{Sn}$ reaction by employing Eq. (12). The experimental elastic scattering probabilities at the backward angle are from Ref. [29]. The reaction cross sections extracted from the experimental elastic scattering angular distribution with optical potential are presented by squares [29].

Figure 12. (Color online) The same as in Fig. 11 but for the ${ }^{4} \mathrm{He}+{ }^{120} \mathrm{Sn}$ reaction. 

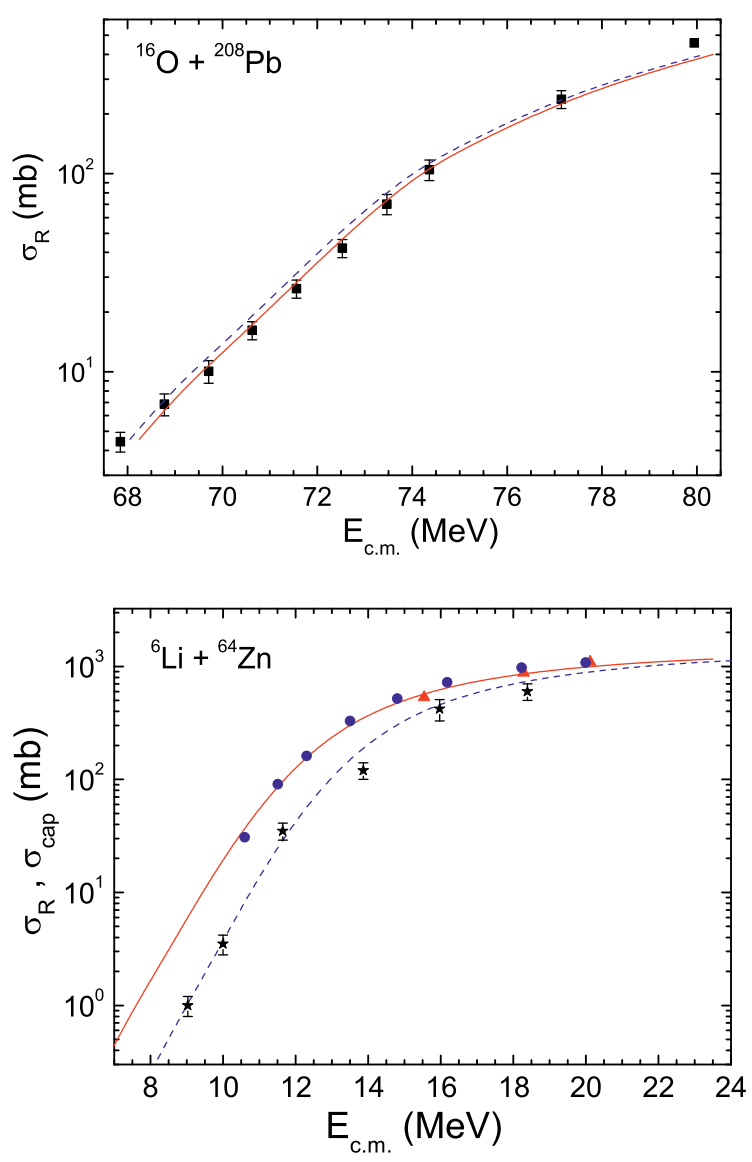

Figure 13. (Color online) The extracted reaction cross sections (solid line) for the ${ }^{16} \mathrm{O}+{ }^{208} \mathrm{~Pb}$ reaction by employing Eq. (12). The experimental elastic scattering probabilities at the backward angle are from Ref. [30]. The reaction cross sections extracted from the experimental elastic scattering angular distribution with optical potential are presented by squares [30].

Figure 14. (Color online) The extracted reaction (solid line) and capture (dashed line) cross sections for the ${ }^{6} \mathrm{Li}+{ }^{64} \mathrm{Zn}$ reaction by employing Eqs. (12) and (13). The experimental elastic and quasi-elastic backscattering probabilities are from Refs. [34, 35]. The reaction cross sections extracted from the experimental elastic scattering angular distribution with optical potential and capture (fusion) cross sections are presented by circles [34, 35], triangles [36, 37] and stars $[34,35]$, respectively.

for ${ }^{7} \mathrm{Li}+{ }^{64} \mathrm{Zn}$. Actually, in Fig. 16, one can observe that our calculations show that

$$
\sigma\left({ }^{7} \mathrm{Li}+{ }^{64} \mathrm{Zn}\right)>\sigma\left({ }^{6} \mathrm{Li}+{ }^{64} \mathrm{Zn}\right),
$$

where

$$
\sigma=\sigma_{R}-\sigma_{c a p} \approx \sigma_{t r}+\sigma_{\text {in }}
$$

since $\sigma_{t r}+\sigma_{i n} \gg \sigma_{B U}$ for these light systems at energies close and below the Coulomb barrier ( $\sigma_{t r}$, $\sigma_{i n}$, and $\sigma_{B U}$ are the transfer, inelastic scattering, and breakup cross sections, respectively). So, our present method of extracting reaction and capture cross sections from backward elastic scattering data allows the approximate determination of the sum of transfer and inelastic scattering cross sections or $\sigma_{t r}+\sigma_{i n}+\sigma_{B U}$ in systems where $P_{B U}$ cannot be neglected. For both systems investigated, the values of these cross sections are shown to increase with $E_{\text {c.m. }}$, reach a maximum slightly above the Coulomb barrier energy and decrease after that. The difference between the two curves in Fig. 16 may be considered approximately as the difference of $\sigma_{t r}$ between the two systems, since $\sigma_{i n}$ should be similar for both systems with the same target, apart from the excitation of the bound excited state of ${ }^{7} \mathrm{Li}$. Because $\sigma_{t r}\left({ }^{7} \mathrm{Li}+{ }^{64} \mathrm{Zn}\right) \gg \sigma_{t r}\left({ }^{6} \mathrm{Li}+{ }^{64} \mathrm{Zn}\right)$, one can find

$$
\sigma_{t r}\left({ }^{7} \mathrm{Li}+{ }^{64} \mathrm{Zn}\right) \approx \sigma\left({ }^{7} \mathrm{Li}+{ }^{64} \mathrm{Zn}\right)-\sigma\left({ }^{6} \mathrm{Li}+{ }^{64} \mathrm{Zn}\right) .
$$



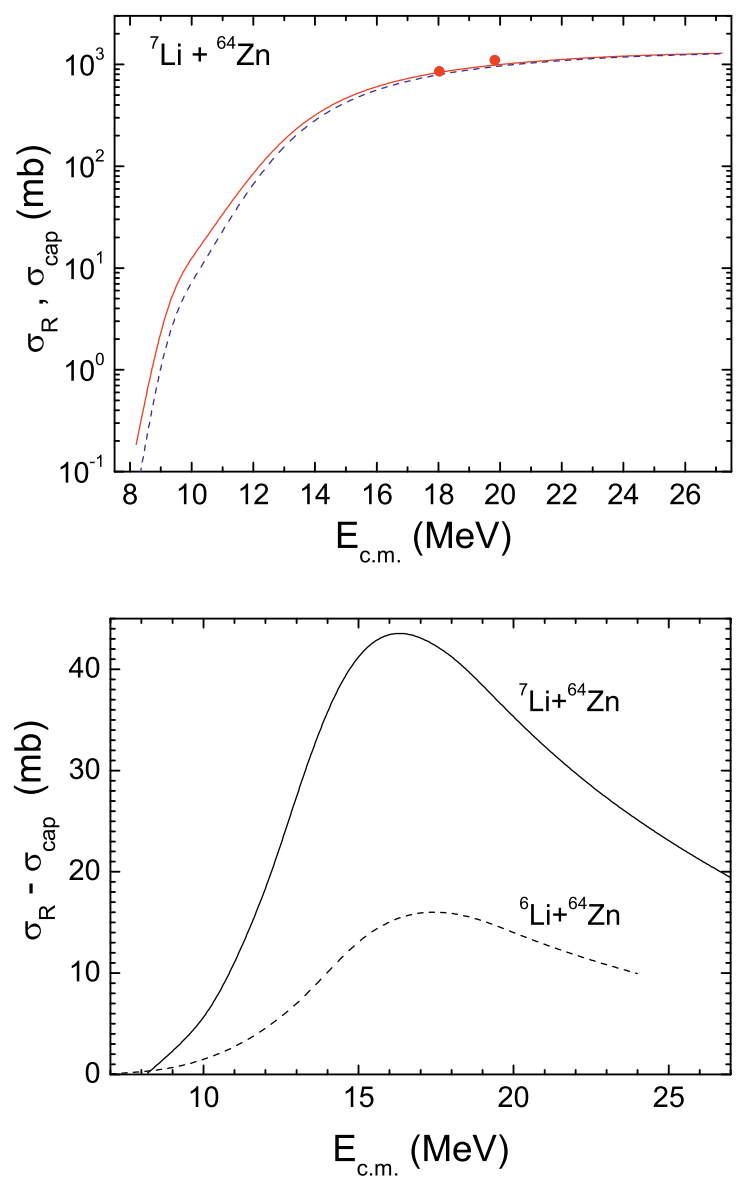

Figure 15. (Color online) The same as in Fig. 14, but for the ${ }^{7} \mathrm{Li}+{ }^{64} \mathrm{Zn}$ reaction. The reaction cross sections extracted from the experimental elastic scattering angular distribution with optical potential are presented by circles $[36,37]$.

Figure 16. The extracted $\sigma_{R}-\sigma_{\text {cap }}$ for the reactions ${ }^{6} \mathrm{Li}+{ }^{64} \mathrm{Zn}$ (dashed line) and ${ }^{7} \mathrm{Li}+{ }^{64} \mathrm{Zn}$ (solid line).

The maximum absolute value of the transfer cross section $\sigma_{t r}$ at energies near the Coulomb barrier is about $30 \mathrm{mb}$. Figure 16 also shows that the difference between transfer cross sections for ${ }^{7} \mathrm{Li}+{ }^{64} \mathrm{Zn}$ and ${ }^{6} \mathrm{Li}+{ }^{64} \mathrm{Zn}$ are much more important than the possible larger $\sigma_{B U}$ for ${ }^{6} \mathrm{Li}$ than for ${ }^{7} \mathrm{Li}$.

\section{Summary}

We propose a new and very simple way to determine reaction and capture (fusion) cross sections, through the relation (12) between the elastic backscattering excitation function and reaction cross section and through the relation (13) between the quasi-elastic scattering excitation function at the backward angle and capture cross section. We show, for several systems, that these methods work well and that the elastic and quasi-elastic backscattering technique could be used as an important and simple tools in the study of the reaction and capture cross sections in the reactions with tightly and weakly bound nuclei. The extraction of reaction (capture) cross sections from the elastic (quasielastic) backscattering is possible with reasonable uncertainties as long as the deviation between the elastic (quasi-elastic) scattering cross section and the Rutherford cross section exceeds the experimental uncertainties significantly. By employing the quasi-elastic backscattering data, one can extract the moments of the angular momentum of the captured system. The behavior of the transfer plus inelas- 
tic excitation function extracted from the experimental probabilities of the elastic and quasi-elastic scatterings at the backward angle was also shown.

We thank H.Q. Zhang for fruitful discussions and suggestions. We are grateful to G. Kiss, R. Lichtenthäler, C.J. Lin, P. Mohr, E. Piasecki, M. Zadro, and H.Q. Zhang for providing us the experimental data. P.R.S.G. acknowledges partial financial support from the CNPq and FAPERJ. This work was supported by DFG, NSFC, RFBR, and JINR grants. The IN2P3(France)-JINR(Dubna) and Polish - JINR(Dubna) Cooperation Programmes are gratefully acknowledged.

\section{References}

[1] V.V. Sargsyan, G.G. Adamian, N.V. Antonenko, and P.R.S. Gomes, Phys. Rev. C 87, 044611 (2013).

[2] V.V. Sargsyan, G.G. Adamian, N.V. Antonenko, and P.R.S. Gomes, Phys. Rev. C 88, 044606 (2013).

[3] V.V. Sargsyan et al., Eur. Phys. J. A 49, 19 (2013).

[4] L.F. Canto, P.R.S. Gomes, R. Donangelo, and M.S. Hussein, Phys. Rep. 424, 1 (2006).

[5] H. Timmers et al., Nucl. Phys. A584, 190 (1995).

[6] H. Timmers et al., J. Phys. G 23, 1175 (1997).

[7] H. Timmers, Ph.D. thesis, Australian National University (1996).

[8] H.Q. Zhang, F. Yang, C. Lin, Z. Liu, and Y. Hu, Phys. Rev. C 57, R1047 (1998).

[9] G.G. Adamian et al., Int. J. Mod. Phys. E 5, 191 (1996); V.V. Sargsyan et al., Phys. Phys. C 84, 064614 (2011).

[10] S. Sinha et al., Phys. Rev. C 64, 024607 (2001).

[11] P. Jacobs, Z. Fraenkel, G. Mamane, and L. Tserruya, Phys. Lett. B 175, 271 (1986).

[12] C.R. Morton et al., Phys. Rev. C 60, 044608 (1999).

[13] Yu.Ts. Oganessian et al., JINR Rapid Commun. 75, 123 (1996).

[14] S.P. Tretyakova et al., Nucl. Phys. A734, E33 (2004).

[15] M. Dasgupta et al., Phys. Rev. Lett. 99, 192701 (2007).

[16] D.E. DiGregorio et al., Phys. Rev. C 39, 516 (1989).

[17] J.R. Leigh et al., Phys. Rev. C 52, 3151 (1995).

[18] E. Piasecki et al., Phys. Rev. C 85, 054608 (2012).

[19] F. Yang et al., Phys. Rev. C 77, 014601 (2008).

[20] H.Q. Zhang et al., Phys. Rev. C 82, 054609 (2010).

[21] H.M. Jia et al., Phys. Rev. C 82, 027602 (2010).

[22] C.J. Lin et al., Nucl. Phys. A787, 281c (2007).

[23] V.V. Sargsyan et al., Phys. Rev. C 86, 054610 (2012).

[24] Y.W. Wu et al., Phys. Rev. C 68, 044605 (2003).

[25] P.R.S. Gomes, J. Lubian, and L.F. Canto, Phys. Rev. C 79, 027606 (2009); P.R.S. Gomes et al., Phys. Rev. C 84, 014615 (2011).

[26] R. Vandenbosch, Annu. Rev. Nucl. Part. Sci. 42, 447 (1992).

[27] S. Gil et al., Phys. Rev. C 43, 701 (1991).

[28] R. Vandenbosch et al., Phys. Rev. C 28, 1161 (1983). 
[29] P. Mohr et al., Phys. Rev. C 82, 047601 (2010).

[30] J.S. Lilley et al., Nucl. Phys. A 463, 710 (1987).

[31] M. Miller et al., Z. Phys. A 300, 97 (1981).

[32] G.G. Kiss et al., Phys. Rev. C 83, 065807 (2011).

[33] I. Badawy et al., Phys. Rev. C 17, 978 (1978).

[34] D. Torresi et al., Eur. Phys. J. Conf 17, 16018 (2011).

[35] A. Di Pietro et al., Phys. Rev. C 87, 064614 (2013)

[36] P.R.S. Gomes et al., Phys. Rev. C 71, 034608 (2005).

[37] P.R.S. Gomes et al., Phys. Lett. B 601, 20 (2004) 